EDITORIAL

\title{
IMMUNOMODULATORS OF HELMINTHES: PROMISING THERAPEUTICS FOR AUTOIMMUNE DISORDERS AND ALLERGIC DISEASES
}

\author{
M V R Reddy \\ Department of Biochemistry \& JB Tropical Disease Research Centre, Mahatma Gandhi Institute of Medical Sciences, \\ Sevagram- 442102, India
}

Human immune system can mount an appropriate immune response against dangerous pathogens and neglect innocent and familiar ones, due to priming of immune system with exposure to various infectious agents since childhood. Westernized countries are facing alarming increase in childhood allergic conditions like rhinitis, atopic dermatitis and asthma (1), inflammatory bowel diseases (IBD) like Crohn's disease and ulcerative colitis and autoimmune disorders like type 1 diabetes, multiple sclerosis and rheumatoid arthritis (2). In contrast, several autoimmune disorders have reduced incidence and severity in geographical regions with high parasite load (3). The low prevalence of infections in developed countries is due to improved health care and personal hygiene and minimal exposure to microorganisms may lead to insufficient stimulation of the immune system of the inhabitants of these regions. This could lead to the altered programming of immune system which may allow expression of inflammatory molecules, a possible reason behind the increased incidence of autoimmune and allergic diseases in developed countries. A great deal of interest has recently been generated on this concept what is known as 'Hygiene hypothesis' (4). Bringing up children in extremely hygienic environments may have negative impact on their immune development making them susceptible to autoimmune diseases and allergic disorders later in life(5). In particular, interplay between helminth infections and host immune system is shown to result in mutual beneficial environment for survival of both, helminthes and host and in the process control the development of autoimmune diseases (6).

\section{Address for Correspondence :}

\section{Dr. MVR Reddy}

Department of Biochemistry \&

JB Tropical Disease Research Centre,

Mahatma Gandhi Institute of Medical Sciences

Sevagram - 442102, Maharashtra, India

E-mail: reddymvr@gmail.com
Helminth parasites are known to survive for long periods in hosts which is attributed to several factors related to both parasite and host and suppression of host immune response is one important factor. Analysis of human immune response to helmith infection like filariasis has shown reduced proliferation of lymphocytes associated with decreased production of IFN $-\gamma$ and increased secretion of IL-4, IL-10 and antibody isotype $\operatorname{lgG} 4$ (7). As IL-4 is a signature cytokine of Th2 cells and IL-10 is anti-inflammatory cytokine, such an immune response may be characterized as being of Th2 type and indeed, anti-inflammatory immunological phenotype is a characteristic of helminth infection. Therefore, it is possible that induction of immunological environment polarized towards Th2 may provide a situation in which autoimmune diseases which are considered to be Th1 mediated would find it difficult to flourish. At the same time it has also been demonstrated that helminth infections could suppress Th2 biased inflammatory disorders, such as asthma, because there also appears to be an inverse correlation between parasite load and atopy $(8,9)$.

\section{Therapeutic implications}

So there is sufficient evidence to emphasize that helminth infections are driving responses that protect against autoimmune diseases. This has led to the number of studies aiming at identifying suitable helminth molecules as immunomodulators with therapeutic application for these diseases. An excretory-secretory (ES) extract of adult stage Nocardia brasiliensis is shown to inhibit the development of asthma (ovalbumin-hypersensitivity model) in the lungs of mice (10). Another important excretory-secretory molecule, ES-62 is a phosphorylcholine (PC) containing glycoprotein secreted by filarial nematode Acanthocheilonema viteae, which has immunomodulatory properties. Exposure to ES-62 has been shown to prevent initiation of collagen-induced arthritis (CIA) in a murine model and also to suppress progression of established disease (11). Infection with Schistosoma mansoni 
or exposure to eggs from this helminth has inhibitory effect on the development of type 1 diabetes in non obese diabetic (NOD) mice (12). There is evidence to show that Schistosoma japonicum egg antigens can induce the production of CD4+ CD25+T cells, resulting in inhibition of development of asthma (13). Schistosome eggs also could protect mice against TNBS induced colitis (14). A protein of $200 \mathrm{KDa}$ of Ascaris suum viz., PSA-1, which could inhibit eosinophilic airway hyper reactivity in $B A L B / c$ mice has been identified and its potent anti-inflammatory activity is attributed to its ability to stimulate regulatory cytokines in macrophages thus leading to the inhibition of pro-inflammatory cytokine production(15). Protease inhibitors such as cystatin and serpin of filarial nematode have been shown to downregulate lymphocyte $T$ cell proliferation of host and to induce anti-inflammatory cytokine response (16).

The positive results in demonstrating the potential of therapeutic effect of worms or their molecules in animals have led to several human studies exploring harmless helminthes like Trichuris suis, the pig whipworm that naturally infects pigs and well tolerated by pig farmers. Treatment of colitis patients with ova of $T$. suis has given promising results and worm-based therapies are under development at several pharmaceutical companies (17). Several large human trials have now been initiated using T.suis eggs on patiets of multiple sclerosis, Chron's diseases and hay fever. The haematophagous hookworm Necator americanus has also been tested for using as alternative worm to treat patients suffering from Crohn's disease and inoculation with this worm is proved to be safe, even in immune suppressed patients (18).

By identifying the way in which helminthes modulates immune response and prevent autoimmune disease, it should be possible to design novel therapeutic approach that may not require infection with live worms. So efforts are being made to develop molecules that mimic the activity of helminthes modulators to develop worm based drugs. Thus exploitation of the ability of chronic helminth infections to modulate host immune system is well poised to be a novel approach in the future to discover safe and effective drugs for the the treatment of chronic inflammatory and allergic diseases.

\section{REFERENCES}

1. Patel SP, Jarvelin MR, Little MP. Systematic review of worldwide variations of the prevalence of wheezing symptoms in children. Environ Health 2008; 7:57.
2. Weinstock JV, Elliott DE. Helminths and the IBD hygiene hypothesis. Inflamm Bowel Dis 2009; 15:128-33.

3. Sewell DL, Reinke EK, Hogan LH, Sandor M, Fabry Z. Immunoregulation of CNS autoimmunity by helminth and mycobacterial infections. Immunol Lett 2002; 82: 101-10.

4. Wills-Karp M, Santeliz J, Karp CL. The germless theory of allergic disease: revisiting the hygiene hypothesis. NatRevImmunol 2001; 1: 69-75.

5. Weinstock JV, Elliott DE. Helminths and the IBD hygiene hypothesis. Inflamm Bowel Dis 2009; 15:128-33.

6. Maizels RM, Pearce EJ, Artis D, Yazdanbakhsh M, Wynn TA. Regulation of pathogenesis and immunity in helminth infections. J Exp Med 2009; 206 (10): 2059-66.

7. Nutman TB, Kumaraswami V. Regulation of the immune response in lymphatic filariasis: perspectives on acute and chronic infection with Wuchereria bancrofti in South India. Parasit Immunol (2001); 28: 389-99.

8. Dunne DW, Cooke A. A worm's eye view of the immune system: Consequences for evolution of human autoimmune disease. Nat Rev Immunol 2005; 5: 420-6.

9. Maizels RM. Infections and allergy: Helminths, hygiene and host immune regulation. Curr Opin Immunol 2005; 17: 656-61.

10. Trujillo-Vargas CM, Werner-Klein M, Wohlleben G, Polte T, Hansen G, Ehlers S, Erb KJ. Helminth-derived products inhibit the development of allergic responses in mice. Am J Respir Crit Care Med 2007; 175(4):336-44.

11. Harnett W, Mclnnes IB, Harnett MM. ES-62, a filarial nematodederived immunomodulator with anti-inflammatory potential. Immunology Letters 2004; 94 (1-2): 27-33.

12. Zaccone P, Zolta' n Fehe' rva' ri1, Jones FM, Sidobre S, Kronenberg M, Dunne DW, Cooke A. Schistosoma mansoni antigens modulate the activity of the innate immune response and prevent onset of type 1 diabetes. Eur J Immunol 2003; 33: 1439-49.

13. Yang J, Zhao J, Yang Y, Zhang L, Yang X, Zhu X, Ji M, Sun N, Su C. Schistosoma japonicum egg antigens stimulate CD4+CD25+T cells and modulate airway inflammation in a murine model of asthma. Immunol 2007; 120: 8-18.

14. Elliott DE, Li J, Blum A, Metwali A, Qadir K, Urban JF Jr, Weinstock $\mathrm{JV}$. Exposure to schistosome eggs protects mice from TNBS induced colitis. AJP-GI 2003; 00049.2002.

15. Oshiro TM, Macedo MS, Macedo-Soares MF. Anti-inflammatory activity of PAS-1, a protein of Ascaris suum. Inflammation Res 2005; 54:17-21

16. Schierack P, Lucius R, Sonnenburg B, Schilling K, Hartmann S. Parasite-Specific Immunomodulatory Functions of Filarial Cystatin. Infection and Immunity 2003; 2422-9.

17. Summers RW, Elliott DE, Urban JF Jr, Thompson RA, Weinstock JV. Trichuris suis therapy for active ulcerative colitis: a randomized controlled trial. Gastroenterol 2005; 128(4): 825-32.

18. Croese J, O'neil J, Masson J, Cooke S, Melrose W, Pritchard D, Speare R. A proof of concept study establishing Necator americanus in Crohn's patients and reservoir donors. Gut 2006; 55(1):136-7. 